OPEN ACCESS

Edited by:

Wei Tao,

Harvard Medical School,

United States

Reviewed by:

Jinzhi Du,

South China University of Technology,

China

Dalong Ni,

University of Wisconsin-Madison,

United States

Yue Pan,

Soochow University, China

*Correspondence:

Lei Zhang

zhanglei646@126.com

Yingjie Xu

xuyingjie@shsmu.edu.cn

Xiaoding Xu

xuxiaod5@mail.sysu.edu.cn

${ }^{t}$ These authors have contributed equally to this work

Specialty section:

This article was submitted to

Experimental Pharmacology and Drug

Discovery,

a section of the journal

Frontiers in Pharmacology

Received: 28 August 2018 Accepted: 28 September 2018

Published: 17 October 2018

Citation:

Saw PE, Zhang A, Nie Y, Zhang L,

$X u Y$ and $X u X(2018)$

Tumor-Associated Fibronectin

Targeted Liposomal Nanoplatform

for Cyclophilin A siRNA Delivery

and Targeted Malignant Glioblastoma

Therapy. Front. Pharmacol. 9:1194.

doi: 10.3389/fphar.2018.01194

\section{Tumor-Associated Fibronectin Targeted Liposomal Nanoplatform for Cyclophilin A siRNA Delivery and Targeted Malignant Glioblastoma Therapy}

\author{
Phei Er Saw ${ }^{1,2 \dagger}$, Ao Zhang ${ }^{1,2 \dagger}$, Yan Nie ${ }^{1,2}$, Lei Zhang ${ }^{1,3 *}$, Yingjie $X u^{4 *}$ and Xiaoding $X u^{1,2 *}$
}

' Guangdong Provincial Key Laboratory of Malignant Tumor Epigenetics and Gene Regulation, Medical Research Center, Sun Yat-sen Memorial Hospital, Sun Yat-sen University, Guangzhou, China, ${ }^{2}$ RNA Biomedical Institute, Sun Yat-sen Memorial Hospital, Sun Yat-sen University, Guangzhou, China, ${ }^{3}$ Department of Hepatobiliary Surgery, Sun Yat-sen Memorial Hospital, Sun Yat-sen University, Guangzhou, China, ${ }^{4}$ Department of Biochemistry and Molecular Cell Biology, Shanghai Key Laboratory for Tumor Microenvironment and Inflammation, Shanghai Jiao Tong University School of Medicine, Shanghai, China

Malignant glioblastoma (GBM) is the most aggressive brain cancer that has a very low survival rate. With the rapid development of nanotechnology in the past few decades, the use of nanoparticles (NPs) for nucleic acid delivery is expected to have a revolutionary impact on GBM therapy. However, clinical success in GBM therapy remains a formidable challenge, mainly due to suboptimal in vivo delivery of therapeutics to glioma cells. Herein, we developed an aptamer-like peptide (aptide)-decorated liposomal nanoplatform for systemic small interfering RNA (siRNA) delivery and targeted GBM therapy. This nanoplatform is mainly composed of the following key components: (i) classic liposome structure with an aqueous core that can encapsulate therapeutic siRNA; (ii) hydrophilic polyethylene glycol (PEG) chains on the outer shell to prolong blood circulation; and (iii) surface-encoded aptide to specifically target the extra-domain $\mathrm{B}$ (EDB) of fibronectin that over-expressed on glioma cells. After systemic administration of these new siRNA delivery NPs, they can target the glioma cells and efficiently inhibit the GBM tumor growth by silencing the expression of cyclophilin A (CypA), which is up-regulated in brain cancer and plays an important role in malignant transformation of brain cancer and maintaining glioma cell stemness. These results suggest that the reported RNA interference (RNAi) NP platform herein could become an effective tool for targeted GBM therapy.

Keywords: nanoparticle, aptide, siRNA, targeted delivery, glioblastoma

\section{INTRODUCTION}

Glioblastoma (GBM) is the most common and aggressive form of brain cancer with poor diagnosis, difficult management, and low survival rate. Even when detected early, less than 5\% of GBM patients are alive 5 years after diagnosis and the median survival rate is around 14.6 months (Behin et al., 2003; Brandsma and van den Bent, 2007; Johnson and O’Neill, 2012). Currently, multimodality treatment approach including surgical resection, radiotherapy, and chemotherapy 
is the standard care for GBM patients (Meyers et al., 2013; Cheng et al., 2014; Mahmoudi and Hadjipanayis, 2014; Krizbai et al., 2016). However, the development of resistance to therapies (e.g., radiotherapy and chemotherapy) has emerged as a persistent clinical problem and ultimately induces the failure of GBM treatment. Multiple reasons have been demonstrated to contribute to this disappointed outcome, including (i) the complex structure of the brain, (ii) the heterogeneous and invasive nature of GBM, and (iii) difficulty in delivering therapeutics specifically to glioma cells (Lesniak and Brem, 2004; Pardridge, 2005; Ozdemir-Kaynak et al., 2018; Shergalis et al., 2018). Therefore, there is a critical need to develop alternative strategies for more effective GBM treatment.

Since the discovery of RNA interference (RNAi) by Fire et al. (1998), RNAi technology has demonstrated significant potential for disease treatment by silencing the expression of target gene(s), especially those encoding "undruggable" proteins (Whitehead et al., 2009; Xu et al., 2016). However, the safe and effective delivery of RNAi agents such as siRNA to target cells remains a major hurdle for the widespread clinical application of RNAi technology. RNAi agents are biomacromolecules with polyanionic characteristics, which are easily attacked by serum nucleases and cannot readily cross cell membrane. Therefore, specific delivery vehicles are required to facilitate the intracellular uptake and cytosolic delivery of RNAi agents (Yang et al., 2012; Kanasty et al., 2013; Liu et al., 2017; Xu et al., 2017a,b). Over the past decades, nanoparticles (NPs) have been demonstrated as a powerful tool toward this end, especially showing hepatocyte-specificity in non-human primates and clinical trials (Zimmermann et al., 2006; Tseng et al., 2009; Jhaveri and Torchilin, 2014; Shi et al., 2017; Durymanov and Reineke, 2018). Nevertheless, it is still challenged to accomplish the systemic delivery of RNAi agents to a particular non-liver diseased tissue (e.g., solid tumor) and cell type, followed by sufficient intra-cytosolic transport. While several RNAi NP platforms have entered into early phase clinical trials for cancer treatment (Zuckerman and Davis, 2015), substantial obstacles still remain, including long blood circulation, selective accumulation at tumor site, and efficient tumor cell internalization. Specifically, these challenges are amplified by the structural complex of the brain (Pardridge, 2005; Mahmoudi and Hadjipanayis, 2014; Shergalis et al., 2018).

To address these issues, we herein developed a liposome-based extra-domain B (EDB)-targeting nanoplatform for systemic siRNA delivery and GBM therapy. As shown in Figure 1, this nanoplatform is composed of a classic liposome structure (i.e., one phospholipid bilayer surrounding an aqueous core), hydrophilic polyethylene glycol (PEG) chains on the outer shell, and surface-encoded aptamer-like peptide (aptide) to specifically target the EDB of fibronectin that over-expressed on glioma cells (Borsi et al., 1992; Castellani et al., 2002; Han and Lu, 2017; Saw et al., 2017). After loading siRNA and then systemic administration, the resulting nanoplatform shows the following unique functions: (i) the hydrophilic PEG chains allow the NPs to escape immunological recognition, thus improving blood circulation; (ii) the surface-encoded aptide moieties can enhance the GBM targeting ability and intracellular siRNA delivery; and (iii) commercial available of the NP compositions and robust NP formulation enables the scale-up of this NP platform. As a proof of concept, we chose cyclophilin A (CypA) as a therapeutic target and systemically evaluated the EDB-targeting NPs for CypA siRNA (siCypA) delivery and its anticancer efficacy. CypA is a ubiquitously distributed protein belonging to the immunophilin family, which shows an activity of peptidylprolyl cis trans isomerase and plays an important role in regulation of protein folding (Wang and Heitman, 2005), trafficking (Shieh et al., 1989; Luban, 1996), assembly (Pan et al., 2008; Tanaka et al., 2011), immune-modulator and cell signaling (Jin et al., 2000; Satoh et al., 2008). It has been demonstrated that CypA is up-regulated in many cancers (e.g., liver, brain, and lung cancers) and is a key determinant for malignant transformation, epithelial to mesenchymal transition (EMT) and cancer metastasis (Yang et al., 2007; Qi et al., 2008). Recent research demonstrated that over-expressed CypA in GBM involves in maintaining glioma cell stemness via $\mathrm{Wnt} / \beta$-catenin signaling pathway (Wang et al., 2017). Our in vivo results show that the systemic delivery of siCypA with the EDB-targeting NP platform can efficiently inhibit CypA expression in the tumor tissue and significantly inhibit GBM tumor growth.

\section{MATERIALS AND METHODS}

\section{Materials}

CypA siRNA (siCypA) and Cy5.5-labeled CypA siRNA (Cy5.5-siCypA) were acquired from Dharmacon (United States). The siRNA sequences are as follows: $5^{\prime}$-UGA CUU CAC ACG CCA UAA UdTdT-3' (sense); 5'-AUU AUG GCG UGU GAA GUC AdTdT-3' (antisense). Protamine sulfate and sepharose CL4B column were purchased from Sigma Aldrich (United States). 1-Palmitoyl-2-oleoyl-sn-glycero-3-phosphocholine (POPC), 1-palmitoyl-2-oleoyl-sn-glycero-3-phospho-(10-rac-glycerol) (POPG), 1,2-distearoyl-sn-glycero-3-phosphoethanolamine (DSPE), and PEG (2000)-DSPE (ammonium salt) (PEG $2000^{-}$ DSPE) were purchased from Avanti Polar Lipids (United States) and used as received. $N$-Maleimide-PEG 2000 -DSPE (ammonium salt) (Mal-PEG $2000-\mathrm{DSPE})$ and plant cholesterol (Chol) were purchased from A.V.T. (Shanghai) Pharmaceuticals (China). EDB-targeting aptide $\left(\mathrm{APT}_{\mathrm{EDB}}\right)$ with an additional cysteine in the $\beta$-hairpin constant loop region (sequence from $N$ to $C$ terminal, CSSPIQGSWTWENGK(C)WTWGIIRLEQ) was synthesized by Guangzhou IGE biotechnology Co., Ltd (China). Lipofectamine 2000 (Lipo2000) was provided by Thermofisher Scientific (United States). Real-time PCR assay kit was procured from Promega (United States). All antibodies were purchased from Abcam (United States) and used according to the manufacturer's protocol. All other chemicals were of reagent grade and used directly.

\section{Methods \\ Synthesis of $A P T_{E D B}$ Conjugated $\mathrm{PEG}_{2000}$-DSPE (APT EDB $-\mathrm{PEG}_{2000}$-DSPE)}

The $\mathrm{APT}_{\mathrm{EDB}}-\mathrm{PEG}_{2000}$-DSPE was synthesized via the reaction between the thiol group of $\mathrm{APT}_{\mathrm{EDB}}$ and maleimide terminal 


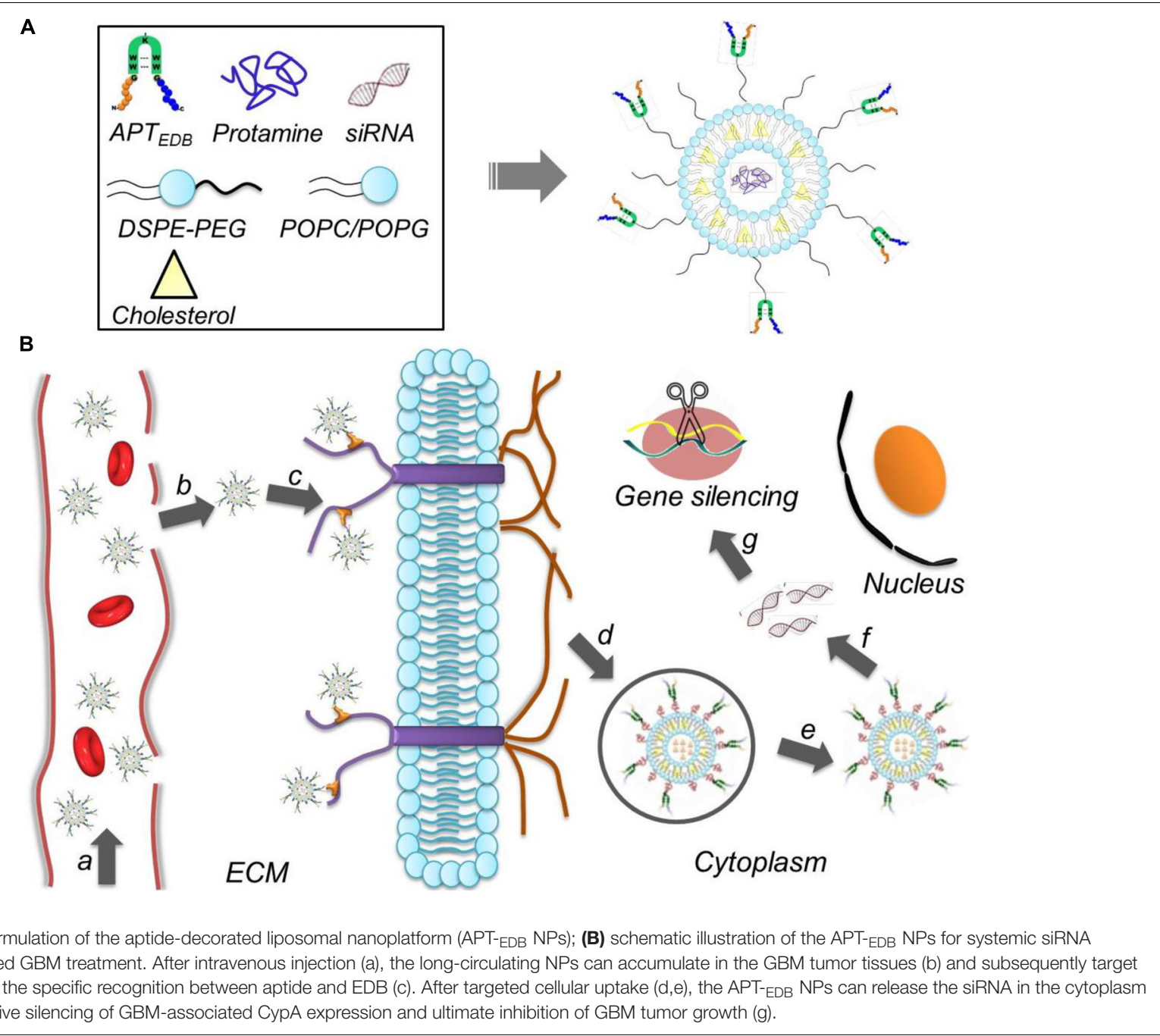

group of Mal- PEG 2000 -DSPE. In brief, $\mathrm{APT}_{\mathrm{EDB}}$ and Mal- PEG $_{2000}$-DSPE were dissolved in dimethyl sulfoxide (DMSO) and chloroform, respectively. Subsequently, these two solutions were mixed in a molar ratio (APT $\mathrm{EDB}_{\mathrm{E}}$ : Mal-PEG $2000-D S P E)$ of 1:2. Under nitrogen atmosphere, the mixture was stirred at room temperature for $12 \mathrm{~h}$. Thereafter, the mixture was transferred to dialysis membrane (MWCO 3500) and dialyzed against deionized water for 3 days. After freeze-drying under vacuum, the $\mathrm{APT}_{\mathrm{EDB}}-\mathrm{PEG}_{2000}-\mathrm{DSPE}$ was collected as a white powder.

\section{Preparation and Characterizations of EDB-Targeting siRNA-Loaded NPs}

The classic rehydration method was employed to prepare the EDB-targeting siRNA-loaded NPs (Saw et al., 2015). POPC, Chol, and POPG were dissolved in chloroform in a molar ratio of 4:3:3 and $\mathrm{APT}_{\mathrm{EDB}}-\mathrm{PEG}_{2000}-\mathrm{DSPE}$ (2.5 wt $\%$ of the total lipid) was then added. The mixture was stirred at room temperature for $10 \mathrm{~min}$ to form a homogenous solution. Subsequently, the solvent was removed by using rotary evaporator and a thin lipid film was thus generated. Then, HEPES-buffered 5\% glucose (HBG) containing siCypA/protamine complexes was added and the resulting mixture $(2 \mathrm{mg} / \mathrm{mL})$ was briefly sonicated to accelerate the formation of siRNA-loaded liposomes. Thereafter, extrusions were performed by using a $100 \mathrm{~nm}$ polycarbonate membrane to ensure the formation of uniform liposomes, which were then passed over sepharose CL-4B column to obtain the purified siRNA-loaded NPs. To prepare the siRNA-loaded NPs without EDB-targeting ability, the POPC, Chol, and POPG were dissolved in chloroform in a molar ratio of 4:3:3 and then the siRNA-loaded NPs were prepared and purified according to the method described above.

The siRNA-loaded NPs were characterized in terms of size, zeta potential, and morphology. The particle size and zeta potential were examined by dynamic light scattering (DLS, Malvern Instruments Corporation). The morphology of siRNA loaded NPs was visualized by transmission electron microscopy (TEM, Tecnai $\mathrm{G}^{2}$ Spirit BioTWIN). To determine the siRNA encapsulation efficiency (EE), Cy5.5-siCypA was used to prepare the siRNA-loaded NPs according to the method aforementioned and the obtained NPs were dispersed in $1 \mathrm{~mL}$ of PBS. Subsequently, a small volume $(5 \mu \mathrm{L})$ of the NP solution was 
withdrawn and mixed with 20-fold DMSO. The standard was prepared by mixing $5 \mu \mathrm{L}$ of naked Cy5.5-siCypA solution with 20-fold DMSO. The fluorescence intensity of Cy5.5-siCypA was measured using a Synergy HT multimode microplate reader (BioTek Instruments), and the siRNA EE\% is calculated as $\mathrm{EE} \%=\left(F I_{N P s} / F I_{\text {Standard }}\right) \times 100$.

\section{In vitro siRNA Release}

Cy5.5-siCypA-loaded NPs were dispersed in $1 \mathrm{~mL}$ of PBS ( $\mathrm{pH}$ 7.4) and then transferred to a Float-a-lyzer G2 dialysis device (MWCO $100 \mathrm{kDa}$, Spectrum) that was immersed in PBS (pH 7.4) at $37^{\circ} \mathrm{C}$. At a predetermined interval, $5 \mu \mathrm{L}$ of the NP solution was withdrawn and mixed with 20-fold DMSO. The fluorescence intensity of Cy5.5-siCypA was determined by Synergy HT multi-mode microplate reader.

\section{Cell Culture}

Human glioma cells (U87MG and U251MG), skin melanoma cells (A375), prostate cancer cells (PC3), breast cancer cells (MCF-7), and Jurkat leukemic T cells were incubated in DMEM medium with $10 \% \mathrm{FBS}$ at $37^{\circ} \mathrm{C}$ in a humidified atmosphere containing $5 \% \mathrm{CO}_{2}$.

\section{Evaluation of the Expression of EDB and CypA in Glioma Cells}

Real-time qPCR was used to evaluate the mRNA level of EDB and CypA in the glioma cells. The intracellular RNA was isolated with RiboEx using an RNA isolation kit (Geneall, South Korea). cDNA was then synthesized using reverse transcription technique using $1 \mu \mathrm{g}$ of total RNA from each sample. The primers used for detecting the EDB domain of fibronectin were $5^{\prime}$-AAC TCA CTG ACC TAA GCT TT-3' (forward) and 5'-CGT TTG TTG TGT CAG TGT AG-3' (reverse). The primers for detecting the CypA were 5'-TAT CTG CAC TGC CAA GAC TGA GTG-3' (forward) and 5'-CTT CTT GCT GGT CTT GCC ATT CC-3' (reverse).

\section{Targeted Cellular Uptake}

Glioma cells (20,000 cells) were seeded in disks and incubated in $2 \mathrm{~mL}$ of DMEM medium containing 10\% FBS for $24 \mathrm{~h}$. Subsequently, the Cy5.5-siCypA-loaded NPs were added at a siRNA concentration of $5 \mathrm{nM}$, and the cells were allowed to incubate at $37^{\circ} \mathrm{C}$ for $4 \mathrm{~h}$. After removing the medium and subsequently washing with PBS buffer thrice, the nuclei were stained with Hoechst 33342 and the cells were then viewed under a FV1000 confocal laser scanning microscope (CLSM, Olympus).

\section{In vitro Gene Silencing}

Glioma cells were seeded in 6-well plates (50,000 cells per well) and incubated in $2 \mathrm{~mL}$ of DMEM medium containing $10 \%$ FBS for $24 \mathrm{~h}$. Subsequently, the cells were incubated with the siCypA-loaded NPs for $24 \mathrm{~h}$. After washing the cells with PBS buffer thrice, the cells were further incubated in fresh medium for another $48 \mathrm{~h}$. Thereafter, the cells were digested by trypsin and the intracellular RNA was isolated for real-time qPCR to examine the CypA expression. As a positive control, Lipo2000/siCypA complexes were prepared according to manufacturer's protocol and then incubated with the glioma cells for $4 \mathrm{~h}$. After washing the cells thrice with PBS, the cells were further incubated for another $48 \mathrm{~h}$ and then collected for real-time qPCR analysis.

\section{In vitro Cell Viability Assay}

Glioma cells were seeded in 96-well plates (5,000 cells per well) and incubated in $0.1 \mathrm{~mL}$ of DMEM medium with $10 \%$ FBS for $24 \mathrm{~h}$. Thereafter, the siCypA-loaded NPs were added at predetermined concentration and the cells were allowed to incubate for $48 \mathrm{~h}$. After removing the medium and washing the cells with PBS thrice, the cell viability was measured using the AlamarBlue assay according to the manufacturer's protocol.

\section{Animals}

Healthy female BALB/c normal mice and nude mice (4-5 weeks old) were purchased from Sun Yat-sen University experimental animal center. All in vivo studies were performed in accordance with a protocol approved by the Institutional Animal Care and Use Committee at Sun Yat-sen University.

\section{Pharmacokinetics}

Healthy female BALB/c normal mice were randomly divided into three groups $(n=3)$ and given an intravenous injection of either (i) naked Cy5.5-siCypA, (ii) Cy5.5-siCypA-loaded NPs, or (iii) EDB-targeting NPs at a 1 nmol siRNA dose per mouse. At predetermined time intervals, $20 \mu \mathrm{L}$ of blood was withdrawn and the wound was pressed for several seconds to stop bleeding. The fluorescence intensity of Cy5.5-siCypA in the blood was determined by Synergy HT multi-mode microplate reader. The blood circulation half-life $\left(t_{1 / 2}\right)$ was calculated according to previous report (Winter et al., 2013; Xu et al., 2017a).

\section{GBM Xenograft Tumor Model}

The tumor model was constructed by subcutaneous injection with $200 \mu \mathrm{L}$ of glioma cell suspension (1:1 mixture of medium and Matrigel) with a density of $2 \times 10^{6}$ cells $/ \mathrm{mL}$ into the back region of healthy female nude mice. When the volume of the tumor xenograft reached $\sim 100 \mathrm{~mm}^{3}$, the mice were used for the in vivo experiments.

\section{Evaluation of EDB Expression in GBM Xenograft Tumor Model}

The tumor tissues were excised from the GBM xenograft tumor-bearing mice and then sectioned using Leica CM 1950 Research Cyrostat (Leica Biosystems, IL, United States) at a $5 \mu \mathrm{m}$ thickness. Subsequently, the tumor sections were washed in PBS and fixed with $4 \%(w / v)$ paraformaldehyde. After blocking with $2 \%$ bovine serum albumin (BSA) for $1 \mathrm{~h}$, the tumor sections were incubated with anti-BC-1 antibody (EDB specific antibody) overnight at $4^{\circ} \mathrm{C}$. Then, the tumor sections were washed in PBS and Alexa Fluor 594-conjugated goat anti-mouse IgG was added. After $1 \mathrm{~h}$ incubation at room temperature, the tumor sections were washed with PBS thrice and mounted with DAPI containing mounting medium. The tumor 
sections were finally viewed under CLSM to examine the EDB expression.

\section{Inhibition of Tumor Growth}

GBM xenograft tumor-bearing nude mice were randomly divided into three groups $(n=4)$ and intravenously injected with (i) PBS, (ii) siCypA-loaded NPs or (iii) EDB-targeting NPs at a 1 nmol siRNA dose per mouse once every two days. All the mice were administrated four consecutive injections and the tumor growth was monitored every two days by measuring perpendicular diameters using a caliper and tumor volume was calculated as follows:

$$
\mathrm{V}=\mathrm{W}^{2} \times \mathrm{L} / 2
$$

where $\mathrm{W}$ and $\mathrm{L}$ are the shortest and longest diameters, respectively.

\section{Histology}

After the aforementioned treatment, the mice in each group were sacrificed at end of the evaluation period, and the tumor tissues and main organs (heart, liver, spleen, lung, and kidney) were collected. After fixing with $4 \%$ paraformaldehyde and then embedding in paraffin, the tissue was sectioned and stained with hematoxylin-eosin (H\&E) and then viewed under an optical microscope. In addition, the apoptosis in the tumor tissues was examined by terminal deoxynucleotidyl transferase dUTP nick-end labeling (TUNEL) assay according to manufacturer's protocol.

\section{RESULTS AND DISCUSSION}

\section{Preparation and Characterizations of the NPs}

EDB of fibronectin has been demonstrated to be highly expressed in the aggressive and malignant GBM (Borsi et al., 1992; Castellani et al., 2002). Therefore, EDB-targeting nanoplatform could be used a robust vehicle to deliver various therapeutics for GBM treatment. Arising from the excellent targeting ability of aptamer, we previously developed aptamer-like peptide (aptide) and demonstrated its strong ability to specifically bind $\mathrm{EDB}$ with high affinity $\left(K_{\mathrm{d}} \sim 16 \mathrm{nM}\right)$ (Saw et al., 2013, 2015, 2017). Based on the high EDB expression in GBM and strong targeting ability of aptide, we herein conjugated the EDB-targeting aptide (APT-EDB) to $\mathrm{PEG}_{2000}$-DSPE, which was then formulated with other lipids (POPC, Chol, and POPG) to obtain the APT-EDB-decorated liposomes (denoted APT-EDB NPs) for the systemic siRNA delivery and targeted GBM treatment (Figure 1). In this work, we employed this nanoplatform to deliver siCypA because it can specifically silence the expression of cancer-associated CypA that over-expressed in GBM (Yang et al., 2007; Qi et al., 2008).
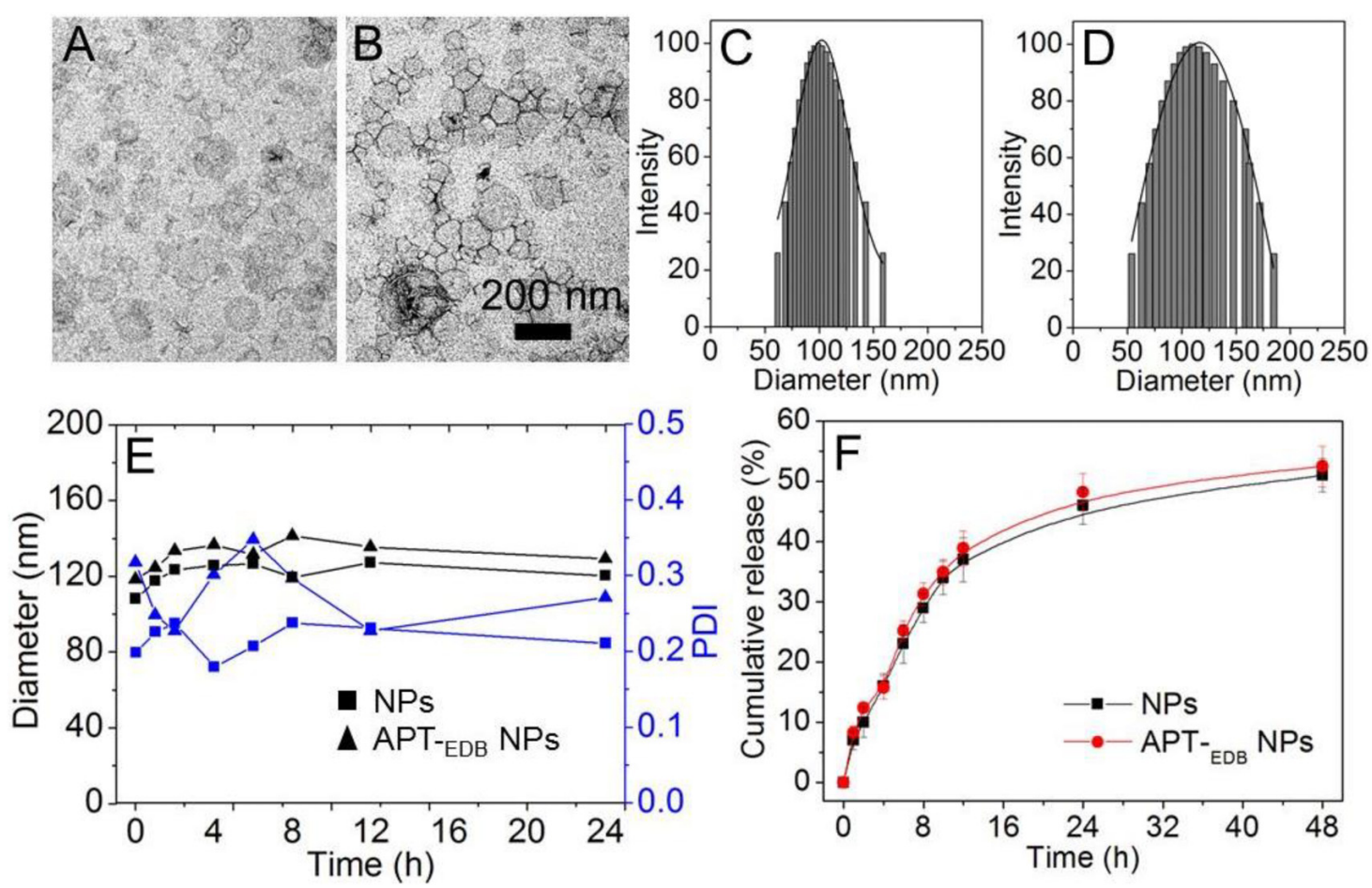

FIGURE 2 | (A-D) TEM images (A,B) and size distribution (C,D) of the siCypA-loaded NPs without aptide decoration (A,C) and the APT-EDB NPS (B,D). (E) Size and polydispersity (PDI) of the siCypA-loaded NPs with (APT-EDB NPS) or without aptide decoration (NPS) incubated in PBS buffer containing $10 \%$ FBS for different time. (F) Cumulative siRNA release from the Cy5.5-siCypA-loaded NPs with (APT-EDB NPs) or without aptide decoration (NPs) incubated in PBS buffer at $37^{\circ} \mathrm{C}$. 

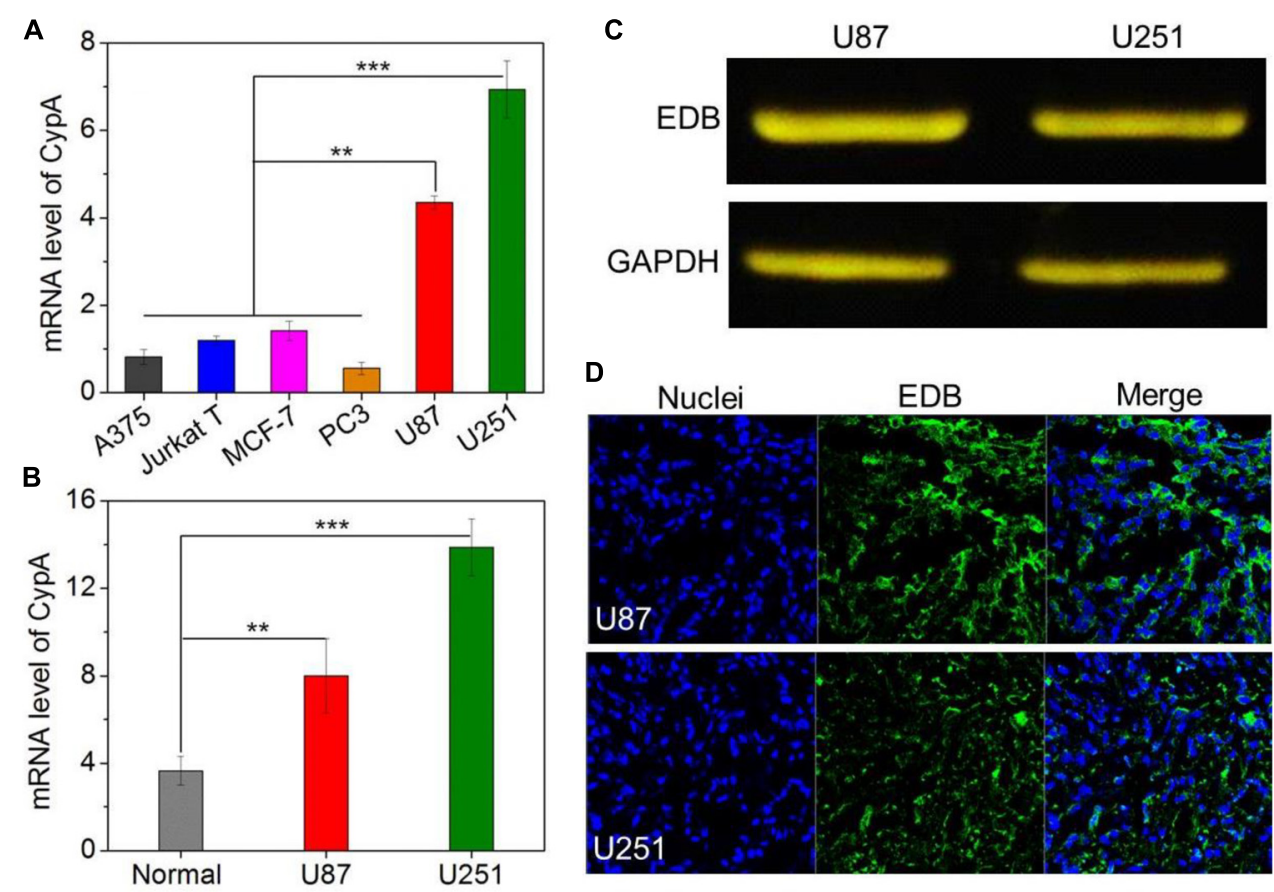

FIGURE 3 | (A) The mRNA level of CypA in the skin melanoma cells (A375), Jurkat leukemic T cells, prostate cancer cells (PC3), breast cancer cells (MCF-7), and glioma cells (U87 and U251). (B) The mRNA level of CypA in the normal brain tissues and GBM xenograft tumor tissues of nude mice. (C) Western blot analysis of the EDB expression in the glioma cells (U87 and U251). (D) Immunofluorescence analysis of the EDB expression in the GBM xenograft tumor tissues of nude mice. The nuclei and EDB were stained with blue and green fluorescence, respectively. ${ }^{* *} P<0.01 ;{ }^{* * *} P<0.001$.
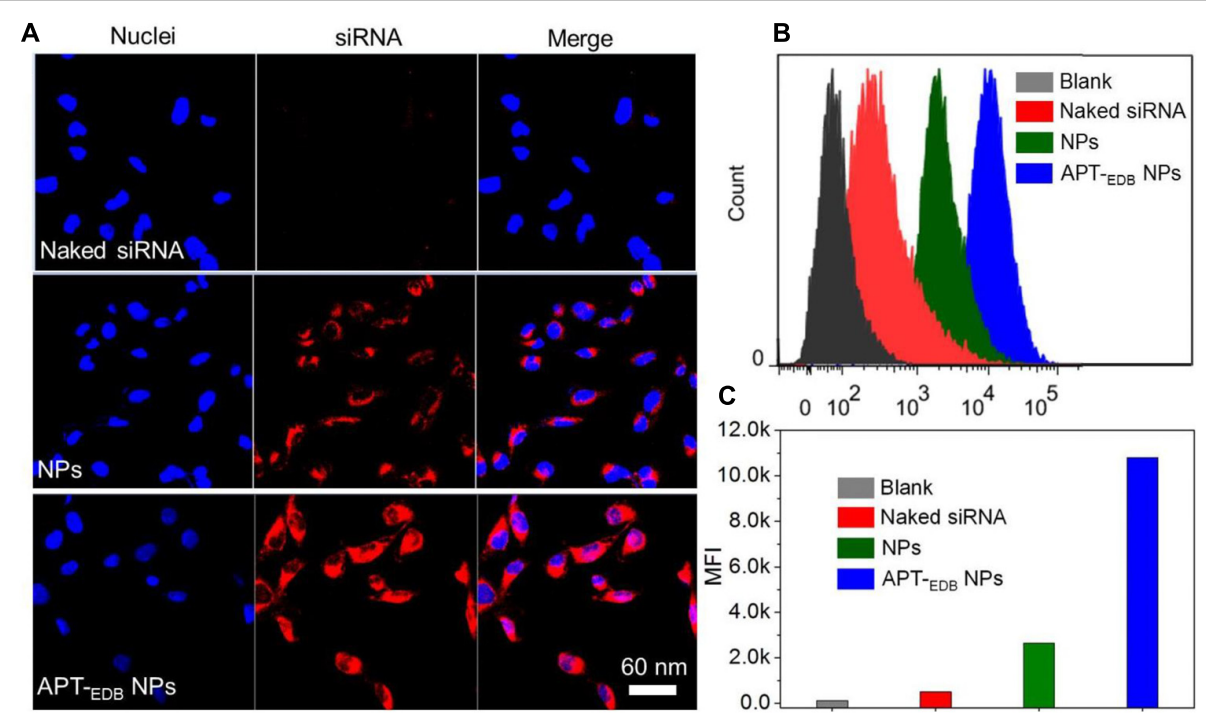

FIGURE 4 | (A) Fluorescent images of U251 cells incubated with the Cy5.5-siCypA-loaded NPs with (APT-EDB NPs) or without aptide decoration (NPs) for 4 h. The nuclei and siRNA were labeled with blue and red fluorescence, respectively. (B,C) Flow cytometry analysis (B) and mean fluorescence intensity [MFI, (C)] of U251 cells incubated with the Cy5.5-siCypA-loaded NPs with (APT-EDB NPs) or without aptide decoration (NPs) for 4 h.

Figure 2 shows the morphology of the siCypA-loaded APT-eDB NPs. Similar as other reported liposomes (Saw et al., 2013, 2015, 2017), the APT-EDB NPs show a spherical morphology with an average size of $\sim 112 \mathrm{~nm}$ determined by DLS (Figure 2D). Compared to the NPs without APT-EDB decoration $(\sim 104 \mathrm{~nm}$, Figure $2 \mathrm{C})$, there is around $10 \mathrm{~nm}$ increase after APT-EDB decoration, suggesting the success in the $\mathrm{APT}_{\mathrm{EDB}}$ decoration because APT-EDB shows a hydrodynamic size of $\sim 5 \mathrm{~nm}$ (Kim et al., 2012). Moreover, due to the presence of PEG chains on the outer layer, these siRNA-loaded NPs 
show a high stability. As shown in Figure 2E, either with or without APT-EDB decoration, possibly due to the presence of weak interaction between the particles and protein, there is a slight change in the particle size and polydispersity density (PDI) when incubating the NPs in $10 \%$ FBS-containing PBS solution for $8 \mathrm{~h}$. However, this interaction will become stable as the incubation time increases and therefore there is no obvious size change after $8 \mathrm{~h}$ incubation. After obtaining these stable NPs, we next labeled the siCypA with fluorescent dye of Cy5.5 to examine the siRNA encapsulation efficiency (EE\%) and release behavior. Through analyzing the fluorescence intensity, the EE was determined as $\sim 90 \%$. The siRNA release profile is shown in Figure 2F. As can be seen, the NPs show a sustained siRNA release behavior. Around $35 \%$ of loaded siRNA can be released within $12 \mathrm{~h}$ and the cumulative release reaches $\sim 50 \% 48$ h later.

\section{Determination of EDB and CypA Expression}

The main purpose of this work is to develop EDB-targeting siRNA delivery nanoplatform for GBM treatment by silencing the CypA expression. Prior to evaluating the gene silencing efficacy of the APT-EDB NPs, we first examined the CypA and EDB expression in the glioma cells. We chose different cancer cell lines and examined the corresponding CypA expression using realtime qPCR. As shown in Figure 3A, compared to skin melanoma cells (A375), Jurkat leukemic T cells, prostate cancer cells (PC3), and breast cancer cells (MCF-7), glioma cells (U87 and U251) show a much higher CypA expression. This encouraging result suggests that CypA is organ-specific over-expressed. To further verify this result, we compared the CypA expression in U87 and U251 xenograft tumors with normal brain tissues. As can be seen, the U87 or U251 xenograft tumors show around twofold or fourfold higher CypA expression that that of normal brain tissues (Figure 3B), implying that silencing CypA expression can be used as a potential strategy for GBM treatment.

After validation of the high CypA expression in the glioma cells, we next examined the EDB expression on these cells. As shown in Figure 3C, western blot analysis demonstrates that both U87 and U251 cells have a high EDB expression. Moreover, if using these two cell lines to construct xenograft tumor model, EDB is also highly expressed in the tumor tissues (green fluorescence, Figure 3D). All these results are consistent with previous reports (Yang et al., 2007; Qi et al., 2008) and indicate that EDB is indeed a suitable biomarker for targeted GBM therapy. Combining with results of PCR analysis (Figures 3A,B), because U251 cells show a relatively higher level of CypA and EDB expression compared to U87 cells, we chose U251 cells to evaluate the targeting and gene silencing ability of the APT-EDB NPs.

\section{Evaluation of GBM-Targeting Ability and in vitro Gene Silencing}

The GBM-targeting ability was evaluated by incubating U251 cells with the siCypA-loaded NPs. From the fluorescent images shown in Figure 4A, compared to naked siRNA, higher amount of siRNA can be observed and distributed in the cytoplasm of the
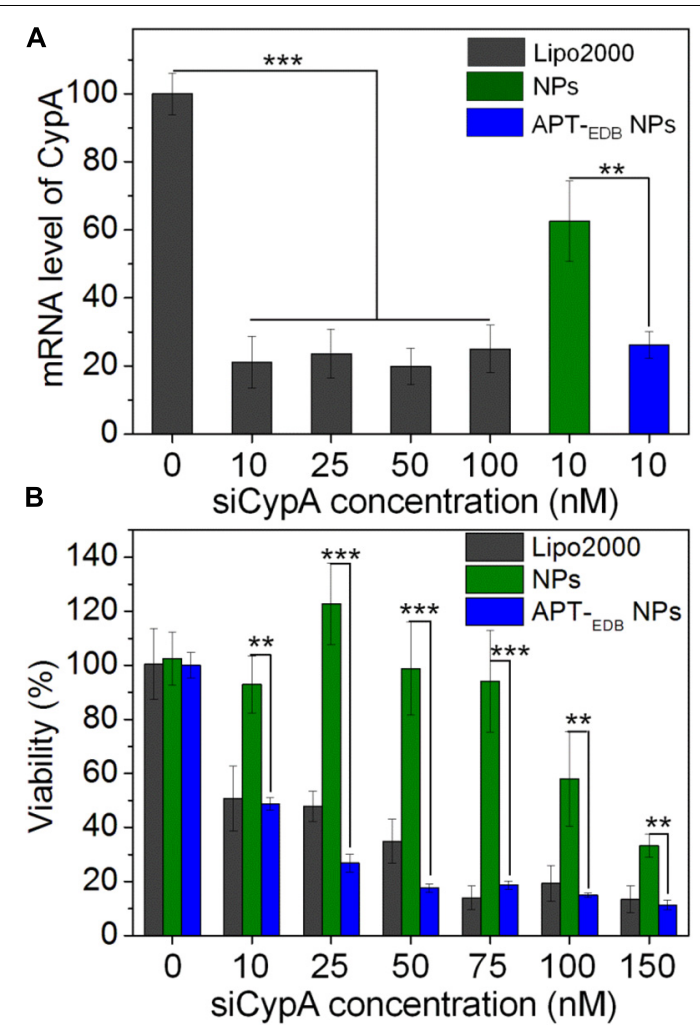

FIGURE 5 | (A) The mRNA level of CypA in U251 cells treated with the Lipo2000/siCypA complexes at various siRNA concentrations, and the siCypA-loaded NPs with (APT-EDB NPS) or without aptide decoration (NPs) at a $10 \mathrm{nM}$ siRNA concentration. (B) Viability of U251 cells treated with the Lipo2000/siCypA complexes, and the siCypA-loaded NPs with (APT-EDB NPs) or without aptide decoration (NPS) at different siRNA concentrations. ${ }^{* *} P<0.01 ;{ }^{* * *} P<0.001$

cells incubated with the siRNA-loaded NPs. More importantly, due to the presence of specific recognition between aptide and EDB, the cellular uptake of APT-EDB NPs is much higher than that of the NPs without aptide decoration. This result is further proven by the flow cytometry analysis. As shown in Figure 4B, U251 cells show stronger ability to internalize the APT-EDB NPs and intracellular mean fluorescence intensity (MFI) is more than fivefold stronger than the cells incubated with the NPs without aptide decoration (Figure 4C).

After validation of the GBM-targeting ability of the APT-EDB NPs, we next examined their in vitro gene silencing efficacy. Figure 5A shows the mRNA level of CypA in U251 cells treated with the siCypA-loaded NPs. With the GBM-targeting ability to improve the cellular uptake, the APT-EDB NPs shows an effective gene silencing and there is around $80 \%$ decrease in the mRNA level of CypA in the cells at a siRNA concentration of $10 \mathrm{nM}$. This high gene silencing efficacy is similar as the commercial available Lipo2000 and is more than twofold higher than that of the NPs without aptide decoration. With this suppressed CypA expression, the cells have a very low viability. As shown in Figure 5B, lower than $50 \%$ of the cells are alive when treated with 

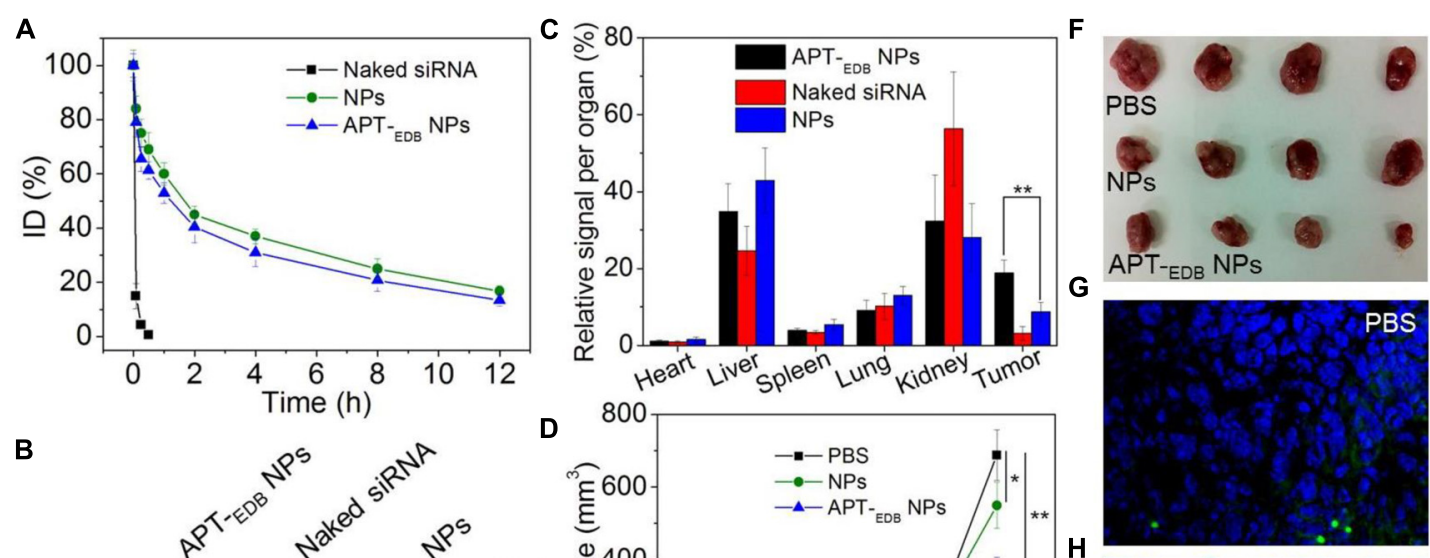

$D$
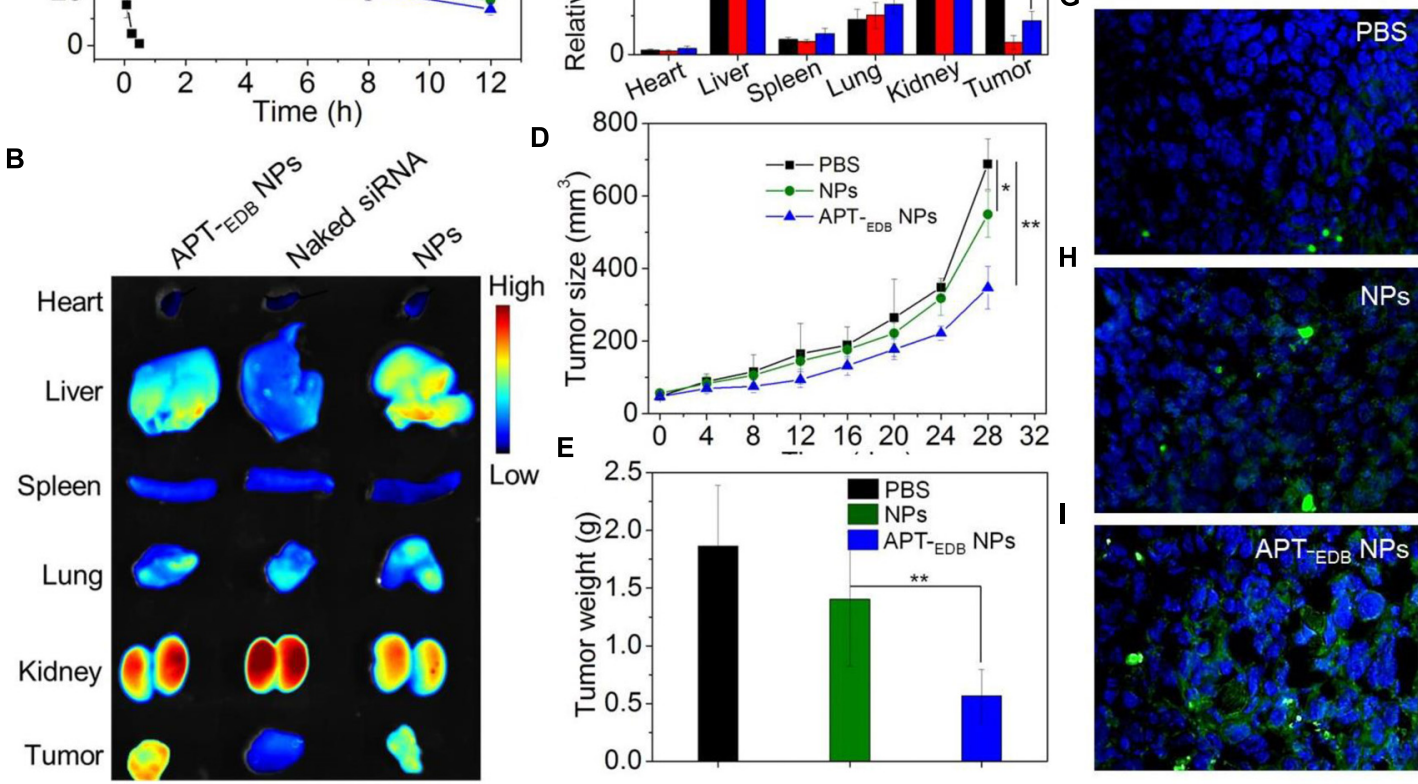

FIGURE 6 | (A) Blood circulation profile of the naked Cy5.5-siCypA and the Cy5.5-siCypA-loaded NPs with (APT-EDB NPs) or without aptide decoration (NPS). (B) Overlaid fluorescent image of the main organs of the GBM xenograft tumor-bearing mice treated with naked siRNA, and the siCypA-loaded NPs with (APT-EDB NPs) or without aptide decoration (NPs). (C) Biodistribution of the siRNA obatined from (B). (D) Tumor growth profile of the GBM xenograft tumor-bearing mice treated with PBS, and the siCypA-loaded NPs with (APT-EDB NPS) or without aptide decoration (NPS). Intravenous injections are indicated by the arrows. (E,F) Weight (E) and representative photograph (F) of the tumor tissues from the mice in each group after 28 day evaluation period. (G-I) TUNEL staining of the collected tumor tissues in (F). TUNEL-positive apoptotic cells were stained with green fluorescence. ${ }^{*} P<0.05 ;{ }^{* *} P<0.01$.

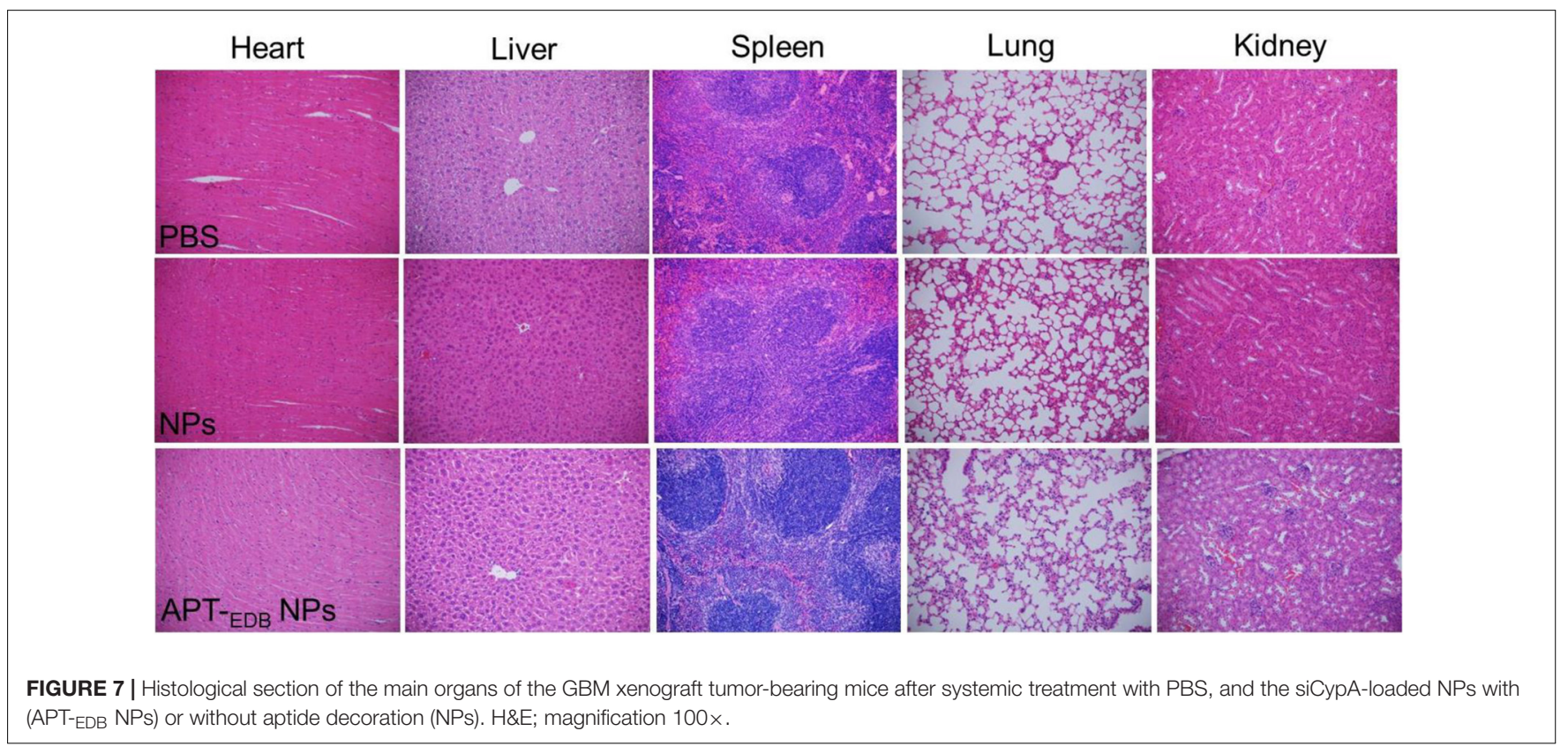


the APT-EDB NPs at a siRNA concentration of $10 \mathrm{nM}$. In contrast, at the same siRNA concentration, around $90 \%$ of the cells are still alive when treated with the NPs without aptide decoration. This result highlights the importance of the GBM-targeting ability of the siRNA-loaded NPs to their gene silencing in glioma cells.

\section{Evaluation of in vivo Anti-tumor Efficacy}

Encouraged by the strong EDB-targeting ability and effective gene silencing of the APT-EDB NPs, we finally evaluated their in vivo anti-tumor efficacy. The pharmacokinetics was first examined by intravenously injecting the Cy5.5-siCypA-loaded NPs to normal adult mice. As shown in Figure 6A, the naked siRNA is rapidly cleared from the blood and its half-life $\left(t_{1 / 2}\right)$ is less than $10 \mathrm{~min}$. In contrast, the APT-EDB NPs show much longer blood circulation with blood $t_{1 / 2}$ of around $4.68 \mathrm{~h}$, which is comparable to the $t_{1 / 2}$ of the NPs without aptide decoration $(\sim 5.12 \mathrm{~h})$. This long circulation feature is mainly attributed to protection by the PEG chains on the outer layer (Knop et al., 2010) and will ensure the accumulation of the APT-EDB NPs in the tumor tissues via enhance and permeable retention (EPR) effect (Bertrand et al., 2014). The biodistribution results in Figures 6B,C also demonstrate our statement. As shown in Figure 6B, the siRNA loaded NPs show higher tumor accumulation than that of naked siRNA. Moreover, due to the presence of APT-EDB targeting ligand, the APT-EDB NPs show more than twofold higher tumor accumulation than that of the NPs without aptide decoration (Figure 6C).

The in vivo anti-tumor efficacy was examined by intravenous injection of the siCypA-loaded APT-EDB NPs to GBM xenograft tumor-bearing mice once every two days at a $1 \mathrm{nmol}$ siRNA dose per mouse. As shown in Figures 6D-F, after four consecutive injections, the tumor growth is obviously inhibited compared to the mice treated with PBS or NPs without aptide decoration. There is around sevenfold increase in the tumor size (from $\sim 50$ to $\sim 350 \mathrm{~mm}^{3}$ ) (Figure 6B). However, for the mice treated with PBS or the NPs without aptide decoration, there is around 14-fold (from $\sim 50$ to $\sim 690 \mathrm{~mm}^{3}$ ) or 11 -fold (from $\sim 50$ to $\sim 550 \mathrm{~mm}^{3}$ ) increase in the tumor size. This tendency is further supported by the result of TUNEL assay. From the images shown in

\section{REFERENCES}

Behin, A., Hoang-Xuan, K., Carpentier, A. F., and Delattre, J. Y. (2003). Primary brain tumours in adults. Lancet 361, 323-331. doi: 10.1016/s0140-6736(03) 12328-8

Bertrand, N., Wu, J., Xu, X., Kamaly, N., and Farokhzad, O. C. (2014). Cancer nanotechnology: the impact of passive and active targeting in the era of modern cancer biology. Adv. Drug Deliv. Rev. 66, 2-25. doi: 10.1016/j.addr.2013.11.009

Borsi, L., Balza, E., Allemanni, G., and Zardi, L. (1992). Differential expression of the fibronectin isoform containing the ED-B oncofetal domain in normal human fibroblast cell lines originating from different tissues. Exp. Cell Res. 199, 98-105. doi: 10.1016/0014-4827(92)90466-L

Brandsma, D., and van den Bent, M. J. (2007). Molecular targeted therapies and chemotherapy in malignant gliomas. Curr. Opin. Oncol. 19, 598-605. doi: 10. 1097/CCO.0b013e3282f0313b

Castellani, P., Borsi, L., Carnemolla, B., Biro, A., Dorcaratto, A., Viale, G. L., et al. (2002). Differentiation between high- and low-grade astrocytoma using a human recombinant antibody to the extra domain-B of fibronectin. Am. J. Pathol. 161, 1695-1700. doi: 10.1016/s0002-9440(10)64446-x
Figures 6G-I, more apoptotic cells can be observed in the tumor section of the mice treated with the siCypA-loaded APT-EDB NPs (Figure 6I). Noting that, the administration of the NPs does not induce apparent in vivo toxicity. As shown in Figure 7, no noticeable histological changes can be found in the tissues from heart, liver, spleen, lung, or kidney of the mice treated with PBS or siRNA-loaded NPs. All these results indicate that the NP platform developed in this work could be potentially used as a safe and efficient gene delivery system for GBM treatment.

\section{CONCLUSION}

In summary, we have developed a robust liposome-based NP platform for systemic siRNA delivery and targeted GBM treatment. This NP platform can target the glioma cells through the specific recognition between their surface-encoded aptide and over-expressed EDB on glioma cells, thereby leading to improved intracellular siRNA delivery and more effective gene silencing in glioma cells. In vivo results show that this long-circulating NP platform can target the GBM tumor tissues and obviously inhibit the tumor growth by silencing the CypA expression. Taken together, the NP platform developed herein could potentially serve as an effective delivery tool for non-invasive GBM treatment.

\section{AUTHOR CONTRIBUTIONS}

PS and XX conceived and designed the experiments. PS, AZ, YN, and YX performed the experiments. PS, YN, LZ, YX, and XX analyzed the data and co-wrote the paper.

\section{FUNDING}

This work was supported by the National Natural Science Foundation of China (81874226 and 81803020) and the Thousand Talents Program for Distinguished Young Scholars.

Cheng, Y., Morshed, R. A., Auffinger, B., Tobias, A. L., and Lesniak, M. S. (2014). Multifunctional nanoparticles for brain tumor imaging and therapy. Adv. Drug Deliv. Rev. 66, 42-57. doi: 10.1016/j.addr.2013.09.006

Durymanov, M., and Reineke, J. (2018). Non-viral Delivery of nucleic acids: insight into mechanisms of overcoming intracellular barriers. Front. Pharmacol. 9:971. doi: 10.3389/fphar.2018.00971

Fire, A., Xu, S., Montgomery, M. K., Kostas, S. A., Driver, S. E., and Mello, C. C. (1998). Potent and specific genetic interference by doublestranded RNA in Caenorhabditis elegans. Nature 391, 806-811. doi: 10.1038/ 35888

Han, Z., and Lu, Z.-R. (2017). Targeting fibronectin for cancer imaging and therapy. J. Mater. Chem. B Mater. Biol. Med. 5, 639-654. doi: 10.1039/C6TB02 008A

Jhaveri, A. M., and Torchilin, V. P. (2014). Multifunctional polymeric micelles for delivery of drugs and siRNA. Front. Pharmacol. 5:77. doi: 10.3389/fphar.2014. 00077

Jin, Z. G., Melaragno, M. G., Liao, D. F., Yan, C., Haendeler, J., Suh, Y. A., et al. (2000). Cyclophilin A is a secreted growth factor induced by oxidative stress. Circ. Res. 87, 789-796. doi: 10.1161/res.87.9.789 
Johnson, D. R., and O'Neill, B. P. (2012). Glioblastoma survival in the United States before and during the temozolomide era. J. Neurooncol. 107, 359-364. doi: 10.1007/s11060-011-0749-4

Kanasty, R., Dorkin, J. R., Vegas, A., and Anderson, D. (2013). Delivery materials for siRNA therapeutics. Nat. Mater. 12, 967-977. doi: 10.1038/nmat3765

Kim, S., Kim, D., Jung, H. H., Lee, I. H., Kim, J. I., Suh, J. Y., et al. (2012). Bio-inspired design and potential biomedical applications of a novel class of high-affinity peptides. Angew. Chem. Int. Ed. Engl. 51, 1890-1894. doi: 10.1002/ anie.201107894

Knop, K., Hoogenboom, R., Fischer, D., and Schubert, U. S. (2010). Poly(ethylene glycol) in drug delivery: pros and cons as well as potential alternatives. Angew. Chem. Int. Ed. Engl. 49, 6288-6308. doi: 10.1002/anie.200902672

Krizbai, I. A., Nyul-Toth, A., Bauer, H. C., Farkas, A. E., Traweger, A., Hasko, J., et al. (2016). Pharmaceutical targeting of the brain. Curr. Pharm. Des. 22, 5442-5462. doi: 10.2174/1381612822666160726144203

Lesniak, M. S., and Brem, H. (2004). Targeted therapy for brain tumours. Nat. Rev. Drug Discov. 3, 499-508. doi: 10.1038/nrd1414

Liu, Y., Xu, C. F., Iqbal, S., Yang, X. Z., and Wang, J. (2017). Responsive nanocarriers as an emerging platform for cascaded delivery of nucleic acids to cancer. Adv. Drug Deliv. Rev. 115, 98-114. doi: 10.1016/j.addr.2017.03.004

Luban, J. (1996). Absconding with the chaperone: essential cyclophilin-Gag interaction in HIV-1 virions. Cell 87, 1157-1159. doi: 10.1016/S0092-8674(00) $81811-5$

Mahmoudi, K., and Hadjipanayis, C. G. (2014). The application of magnetic nanoparticles for the treatment of brain tumors. Front. Chem. 2:109. doi: 10 . 3389/fchem.2014.00109

Meyers, J. D., Doane, T., Burda, C., and Basilion, J. P. (2013). Nanoparticles for imaging and treating brain cancer. Nanomedicine 8, 123-143. doi: 10.2217/ nnm.12.185

Ozdemir-Kaynak, E., Qutub, A. A., and Yesil-Celiktas, O. (2018). Advances in glioblastoma multiforme treatment: new models for nanoparticle therapy. Front. Physiol. 9:170. doi: 10.3389/fphys.2018.00170

Pan, H., Luo, C., Li, R., Qiao, A., Zhang, L., Mines, M., et al. (2008). Cyclophilin $\mathrm{A}$ is required for CXCR4-mediated nuclear export of heterogeneous nuclear ribonucleoprotein $\mathrm{A} 2$, activation and nuclear translocation of ERK1/2, and chemotactic cell migration. J. Biol. Chem. 283, 623-637. doi: 10.1074/jbc. M704934200

Pardridge, W. M. (2005). The blood-brain barrier: bottleneck in brain drug development. NeuroRx 2, 3-14. doi: 10.1602/neurorx.2.1.3

Qi, Y. J., He, Q. Y., Ma, Y. F., Du, Y. W., Liu, G. C., Li, Y. J., et al. (2008). Proteomic identification of malignant transformation-related proteins in esophageal squamous cell carcinoma. J. Cell. Biochem. 104, 1625-1635. doi: $10.1002 / j \mathrm{cb} .21727$

Satoh, K., Matoba, T., Suzuki, J., O’Dell, M. R., Nigro, P., Cui, Z., et al. (2008). Cyclophilin A mediates vascular remodeling by promoting inflammation and vascular smooth muscle cell proliferation. Circulation 117, 3088-3098. doi: 10.1161/CIRCULATIONAHA.107.756106

Saw, P. E., Kim, S., Lee, I.-H., Park, J., Yu, M., Lee, J., et al. (2013). Aptideconjugated liposome targeting tumor-associated fibronectin for glioma therapy. J Mater. Chem. B 1, 4723-4726. doi: 10.1039/C3TB20815J

Saw, P. E., Park, J., Jon, S., and Farokhzad, O. C. (2017). A drug-delivery strategy for overcoming drug resistance in breast cancer through targeting of oncofetal fibronectin. Nanomedicine 13, 713-722. doi: 10.1016/j.nano.2016.10.005

Saw, P. E., Park, J., Lee, E., Ahn, S., Lee, J., Kim, H., et al. (2015). Effect of PEG pairing on the efficiency of cancer-targeting liposomes. Theranostics 5, 746-754. doi: $10.7150 /$ thno. 10732

Shergalis, A., Bankhead, A. III, Luesakul, U., Muangsin, N., and Neamati, N. (2018). Current challenges and opportunities in treating glioblastoma. Pharmacol. Rev. 70, 412-445. doi: 10.1124/pr.117.014944

Shi, J., Kantoff, P. W., Wooster, R., and Farokhzad, O. C. (2017). Cancer nanomedicine: progress, challenges and opportunities. Nat. Rev. Cancer 17, 20-37. doi: $10.1038 /$ nrc. 2016.108
Shieh, B. H., Stamnes, M. A., Seavello, S., Harris, G. L., and Zuker, C. S. (1989). The ninaA gene required for visual transduction in Drosophila encodes a homologue of cyclosporin A-binding protein. Nature 338, 67-70. doi: 10.1038/338067a0

Tanaka, H., Shimazaki, H., Kimura, M., Izuta, H., Tsuruma, K., Shimazawa, M., et al. (2011). Apoptosis-inducing factor and cyclophilin A cotranslocate to the motor neuronal nuclei in amyotrophic lateral sclerosis model mice. CNS Neurosci. Ther. 17, 294-304. doi: 10.1111/j.1755-5949.2010. 00180.x

Tseng, Y. C., Mozumdar, S., and Huang, L. (2009). Lipid-based systemic delivery of siRNA. Adv. Drug Deliv. Rev. 61, 721-731. doi: 10.1016/j.addr.2009.03.003

Wang, G., Shen, J., Sun, J., Jiang, Z., Fan, J., Wang, H., et al. (2017). Cyclophilin A maintains glioma-initiating cell stemness by regulating Wnt/beta-catenin signaling. Clin. Cancer Res. 23, 6640-6649. doi: 10.1158/1078-0432.CCR-170774

Wang, P., and Heitman, J. (2005). The cyclophilins. Genome Biol. 6:226. doi: $10.1186 / \mathrm{gb}-2005-6-7-226$

Whitehead, K. A., Langer, R., and Anderson, D. G. (2009). Knocking down barriers: advances in siRNA delivery. Nat. Rev. Drug Discov. 8, 129-138. doi: 10.1038/ $\operatorname{nrd} 2742$

Winter, H., Ginsberg, A., Egizi, E., Erondu, N., Whitney, K., Pauli, E., et al. (2013). Effect of a high-calorie, high-fat meal on the bioavailability and pharmacokinetics of PA-824 in healthy adult subjects. Antimicrob. Agents Chemother. 57, 5516-5520. doi: 10.1128/aac.00798-13

Xu, X., Saw, P. E., Tao, W., Li, Y., Ji, X., Yu, M., et al. (2017a). Tumor microenvironment-responsive multistaged nanoplatform for systemic RNAi and cancer therapy. Nano Lett. 17, 4427-4435. doi: 10.1021/acs.nanolett. $7 \mathrm{~b} 01571$

Xu, X., Wu, J., Liu, Y., Saw, P. E., Tao, W., Yu, M., et al. (2017b). Multifunctional envelope-type siRNA delivery nanoparticle platform for prostate cancer therapy. ACS Nano 11, 2618-2627. doi: 10.1021/acsnano. $6 \mathrm{~b} 07195$

Xu, X., Wu, J., Liu, Y., Yu, M., Zhao, L., Zhu, X., et al. (2016). Ultra-pH-responsive and tumor-penetrating nanoplatform for targeted siRNA delivery with robust Anti-cancer efficacy. Angew. Chem. Int. Ed. Engl. 55, 7091-7094. doi: 10.1002/ anie. 201601273

Yang, H., Chen, J., Yang, J., Qiao, S., Zhao, S., and Yu, L. (2007). Cyclophilin $\mathrm{A}$ is upregulated in small cell lung cancer and activates ERK1/2 signal. Biochem. Biophys. Res. Commun. 361, 763-767. doi: 10.1016/j.bbrc.2007. 07.085

Yang, X. Z., Du, J. Z., Dou, S., Mao, C. Q., Long, H. Y., and Wang, J. (2012). Sheddable ternary nanoparticles for tumor acidity-targeted siRNA delivery. ACS Nano 6, 771-781. doi: 10.1021/nn204240b

Zimmermann, T. S., Lee, A. C., Akinc, A., Bramlage, B., Bumcrot, D., Fedoruk, M. N., et al. (2006). RNAi-mediated gene silencing in non-human primates. Nature 441, 111-114. doi: 10.1038/nature04688

Zuckerman, J. E., and Davis, M. E. (2015). Clinical experiences with systemically administered siRNA-based therapeutics in cancer. Nat. Rev. Drug Discov. 14, 843-856. doi: $10.1038 / \mathrm{nrd} 4685$

Conflict of Interest Statement: The authors declare that the research was conducted in the absence of any commercial or financial relationships that could be construed as a potential conflict of interest.

The handling Editor declared a past co-authorship with the authors PS and XX.

Copyright (๑) 2018 Saw, Zhang, Nie, Zhang, Xu and Xu. This is an open-access article distributed under the terms of the Creative Commons Attribution License (CC BY). The use, distribution or reproduction in other forums is permitted, provided the original author(s) and the copyright owner(s) are credited and that the original publication in this journal is cited, in accordance with accepted academic practice. No use, distribution or reproduction is permitted which does not comply with these terms. 